\title{
Enhancement of bipolar carrier transport in oligofluorene films through alignment in the liquid-crystalline phase
}

\author{
Li-Yin Chen, Wen-Yi Hung, Yu-Ting Lin, and Chung-Chih Wu ${ }^{\text {a) }}$ \\ Department of Electrical Engineering, Graduate Institute of Electro-optical Engineering, and Graduate \\ Institute of Electronics Engineering, National Taiwan University, Taipei, Taiwan 106, \\ Republic of China
}

Teng-Chih Chao, Tsung-His Hung, and Ken-Tsung Wong ${ }^{\text {b) }}$

Department of Chemistry, National Taiwan University, Taipei, Taiwan 106, Republic of China

(Received 19 May 2005; accepted 28 July 2005; published online 7 September 2005)

\begin{abstract}
We investigate the formation of aligned liquid-crystal glasses of an oligofluorene and perform comparative studies of carrier-transport properties of the oligofluorene in both amorphous films and glassy liquid-crystal films. With mesophase-mediated molecular alignment, the bipolar carrier-transport capability of oligofluorene solid films is enhanced by more than an order of magnitude. High bipolar carrier mobilities, up to $2.0 \times 10^{-2} \mathrm{~cm}^{2} / \mathrm{V} \mathrm{s}$ for holes and up to 2.3 $\times 10^{-2} \mathrm{~cm}^{2} / \mathrm{V} \mathrm{s}$ for electrons, are observed in the aligned glassy liquid-crystal films. (C) 2005 American Institute of Physics. [DOI: 10.1063/1.2042536]
\end{abstract}

Advances in organic optoelectronic devices in recent years, such as organic light-emitting devices, organic solar cells and photodetectors, and organic thin-film transistors, etc., have imposed substantial demands on low-molar-mass organic materials with high mobilities. ${ }^{1,2}$ Investigations reveal that by increasing molecular ordering from amorphous polycrystalline to monocrystalline, carrier mobilities in molecular thin films may be drastically increased due to a larger overlap of wave functions of molecular orbitals. ${ }^{1-3}$ Since the growth of monocrystalline molecular thin films without structural imperfections in general is difficult, uncontrolled crystallization would lead to polycrystalline films with grains and grain boundaries of various sizes. Such structural defects often cause deep carrier trapping, often degrading carrier transport so significantly that carriers could hardly transport. ${ }^{4}$ Furthermore, morphological nonhomogeneity in imperfect crystalline systems may be problematic in some electronic/ optical applications requiring high homogeneity. ${ }^{5}$ Therefore, embedding some ordering in condensed molecular systems, yet still keeping the homogeneous nature of an amorphous state, appears an attractive alternative.

One recent approach of achieving such characteristics and thus acquiring higher mobility in an organic material is to induce molecular ordering through mesophase (liquidcrystal) formation. ${ }^{6-12}$ Several types of liquid-crystalline (LC) phases have been investigated for a range of materials and enhanced time-of-flight mobilities of $10^{-3}-10^{-1} \mathrm{~cm}^{2} / \mathrm{V} \mathrm{s}$ have been reported. ${ }^{6-11}$ Yet except for few cases in LC polymers or photopolymerized LCs, ${ }^{10-12}$ most of these studies, however, were performed in somewhat fluidic mesophases at elevated temperatures. To enhance carrier mobilities in homogeneous molecular solid films with mesophase ordering, it is desired that LC ordering of low-molar-mass materials be stabilized in the glassy state, e.g., forming LC glasses upon cooling from the mesophase at elevated temperatures. However, due to easy crystallization of molecular materials upon

\footnotetext{
a) Author to whom correspondence should be addressed; electronic mail: chungwu@cc.ee.ntu.edu.tw

b)Electronic mail: kenwong@ntu.edu.tw
}

cooling, high mobility obtained in mesophases is lost due to formation of grain boundaries and carrier traps. Therefore, enhancing carrier mobility through mesophase ordering thus far has not been successfully demonstrated for purely molecular solid films. Even in polymeric systems, only the enhancement of hole mobility through LC alignment was reported for solid films. ${ }^{10-12}$

As such, a few recent findings in related characteristics of oligofluorenes are worthy of attention. When their $s p^{3} \mathrm{C} 9$ carbons are substituted with appropriate alkyl chains, oligofluorenes have been shown to exhibit mesophases at elevated temperatures, and yet also the stable glass phase at room temperature. $^{13,14}$ Indeed, it has been demonstrated that liquid-crystal glasses of oligofluorenes could be formed by freezing their mesophases down to room temperature without crystallization. ${ }^{13,14}$ These characteristics, together with our recent findings that oligofluorenes exhibit intriguing nondispersive ambipolar carrier transport and high mobilities in the amorphous state, ${ }^{15,16}$ strongly suggest the possibility of achieving enhanced carrier mobilities (for both holes and electrons) in oligofluorenes solid films through mesophasemediated molecular alignment. In this letter, we investigate the formation of aligned LC glasses of an oligofluorene and perform comparative studies of carrier-transport properties of the oligofluorene in both amorphous films and glassy LC films.

The structure of the oligofluorene $\mathbf{E 4}$ under investigation is shown in Fig. 1. The backbone of $\mathbf{E} 4$ contains four fluorene units and ethyl substituents are introduced on C9 atoms. E4 was synthesized by Suzuki coupling reactions of 7,7'-dibromo-9,9,9' ,9' -tetraethyl-2,2' -bifluorene and 2-(4,4,5,5-tetramethyl-1,3,2-dioxaborolyl)-9,9-diethylfluorene using synthetic protocols reported previously, ${ }^{14-16}$ and the details of syntheses and characterization will be re-

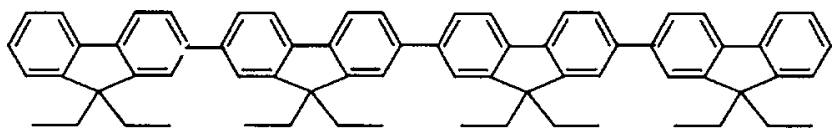

FIG. 1. The molecular structure of E4. 
(a)

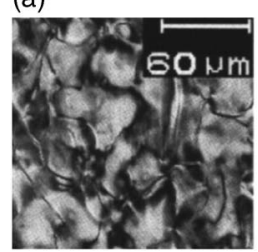

(b)

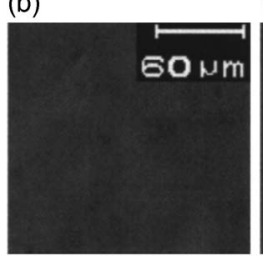

(c)

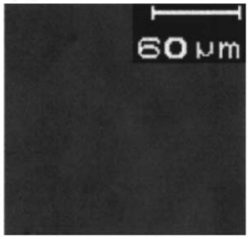

FIG. 2. (a) Photo of a micrometer thick E4 sample (sandwiched between two glass slides) taken under the polarized microscope at $220{ }^{\circ} \mathrm{C}$; (b) photo of an aligned LC cell of $\mathbf{E} 4$ taken under the polarized microscope at $220^{\circ} \mathrm{C}$; (c) photo of the sample in (b) taken after rapidly cooling $\left(\sim-60^{\circ} \mathrm{C} / \mathrm{min}\right)$ to room temperature.

ported elsewhere. For the present purpose, it suffices to mention that their molecular structures were validated with elemental and spectroscopic analyses. Synthesized E4 was subject to sublimation purification before use in subsequent analyses. According to characterization with differential scanning calorimetry, E4 possesses a reasonably high glasstransition temperature $\left(T_{g}\right)$ of $\sim 134{ }^{\circ} \mathrm{C}$. Thus, E4 could form homogeneous and amorphous films through vacuum deposition, and the amorphous nature of vacuum-deposited films are confirmed by x-ray diffraction. When observed under a polarized optical microscope equipped with a heating/ cooling stage, E4 also exhibits a nematic LC phase at 190$230{ }^{\circ} \mathrm{C}$. Figure 2(a) shows the photo of a micrometer thick E4 sample (sandwiched between two glass slides) taken under the polarized microscope at $220^{\circ} \mathrm{C}$, in which the threaded texture typical of nematic LCs is clearly observed. Without applying alignment, the LC cell of $\mathbf{E 4}$ contains small domains and domain boundaries, which hindered observation of nondispersive carrier transport in the mobility measurement. To induce molecular alignment to a larger extent, E4 was sandwiched between two glass substrates coated with polyimide films as alignment layers, using glassrod spacers of $\sim 10 \mu \mathrm{m}$ to control the cell gap. The polyimide film was prepared by first spin coating a polyamic acid precursor on the substrate (either a glass or a glass precoated with a $30 \mathrm{~nm}$ semitransparent Ag film). This precursor film was then baked at $200{ }^{\circ} \mathrm{C}$ for $1 \mathrm{~h}$ to achieve full imidization. The resulting polyimide film of $40 \mathrm{~nm}$ thickness was then rubbed with a dustless cloth for surface alignment. Figure 2(b) shows the photo of an aligned LC cell of $\mathbf{E 4}$ taken under the polarized microscope at $220{ }^{\circ} \mathrm{C}$. Upon alignment, a nearly monodomain uniaxial alignment of $\mathbf{E 4}$ molecules was achieved. By rapidly cooling the aligned sample to room temperature $\left(\sim-60{ }^{\circ} \mathrm{C} / \mathrm{min}\right)$, the uniaxial molecular alignment mediated by fluidic nematic mesomorphism was preserved and a uniaxially aligned and uniform glassy nematic film was obtained, as shown in Fig. 2(c). E4 has a tendency to crystallize at temperatures between the $T_{g}$ and the LC temperature, yet the fast enough cooling rate bypasses crystallization.

Charge transport in E4 films were characterized by the time-of-flight (TOF) transient photocurrent technique. For vacuum-deposited amorphous E4 films, the sample structure used was glass substrate/Ag $(30 \mathrm{~nm}) / \mathbf{E} 4(1.28 \mu \mathrm{m}) / \mathrm{Al}(150$ $\mathrm{nm})$. For the uniaxially aligned glassy nematic film, the sample structure used was glass substrate/Ag (30 nm)/ alignment layer $(40 \mathrm{~nm}) / \mathbf{E 4}(10 \mu \mathrm{m}) /$ alignment layer $(40$ $\mathrm{nm}) / \mathrm{Ag}(30 \mathrm{~nm}) / \mathrm{glass}$ substrate, ${ }^{14-16}$ in which the cell gap was controlled by using glass rods as spacers. Ag and $\mathrm{Al}$ were chosen as blocking contacts for the TOF samples due to Downloaded 17 Feb 2009 to 140.112 .113 .225 . Redistribution subje their poor carrier-injection capability for the present oligofluorene. In the TOF mobility measurement, a frequencytripled Nd: YAG laser (355 nm, 10 ns pulse duration) was used for pulsed illumination through the semitransparent $\mathrm{Ag}$. This wavelength matches the absorption peak of E4( $\sim 355 \mathrm{~nm}$ ) and gives a large absorption coefficient of $>10^{5} \mathrm{~cm}^{-1}$, ensuring the penetration depth of the illumination ( 100-200 nm) and the sheet of photogenerated carriers to be thin enough in comparison with the thickness of the E4 layer. The TOF measurements were performed in a $10^{-5}$ Torr vacuum chamber. Under an applied dc bias, the transient photocurrent was recorded with a digital storage oscilloscope. The intensity of illumination was adjusted to keep the photogenerated charges less than $5 \%$ of the sample capacitor charge, avoiding space-charge-induced nonuniform distribution of the electric field during the drift of carriers. Depending on the polarity of the applied bias, selected carriers (holes or electrons) are swept across the sample with a transit time of $t_{T}$. With the applied bias $V$ and the sample thickness $D$, the applied electric field $E$ is then $V / D$, and the carrier mobility is given by $\mu=D /\left(t_{T} \cdot E\right)=D^{2} /\left(V \cdot t_{T}\right)$.

Both the amorphous film and the aligned glassy nematic film of E4 exhibit nondispersive ambipolar carrier-transport characteristics. Figures 3(a) and 3(b) show representative TOF transients of holes and electrons, respectively, for the amorphous E4 film at room temperature. On the other hand, Figs. 3(c) and 3(d) show representative TOF transients of holes and electrons, respectively, for the aligned glassy nematic film of $\mathbf{E} 4$ at room temperature. In all of these curves, the short initial spike in the transient, limited by the time resolution of the measurement setup, is followed first by a constant-current plateau and then by a drop. The final falloff of the current is caused by the carriers reaching the collection electrode, where they are discharged. The appearance of a plateau indicates that the charge carriers have attained a constant drift velocity and hence nondispersive carrier transport. ${ }^{17-19}$ The carrier transit time $t_{T}$ needed for determining the mobility can be unambiguously evaluated from the intersection point of the asymptotes to the plateau and the tail sections in the double logarithmic representation (insets of Fig. 3). Hole and electron mobilities thus determined for both the amorphous film and the nematic glass film are shown as a function of $E^{1 / 2}$ in Figs. 4(a) and 4(b), respectively. The field-dependence of mobilities in Fig. 4 follows the universal Poole-Frenkel relationship: $\mu \propto \exp \left(\beta E^{1 / 2}\right)$, where $\beta$ is the Poole-Frenkel factor. ${ }^{17-19}$ Such a relationship is often observed in somewhat disordered organic systems and could be attributed to effects of energetic and positional disorder on the hopping conduction in disordered organic solids. $^{17-19}$

The aligned glassy nematic film shows a hole mobility up to $2.0 \times 10^{-2} \mathrm{~cm}^{2} / \mathrm{V} \mathrm{s}$ and an electron mobility up to $2.3 \times 10^{-2} \mathrm{~cm}^{2} / \mathrm{V} \mathrm{s}$ for fields from $1.0 \times 10^{5}$ to 3.0 $\times 10^{5} \mathrm{~V} / \mathrm{cm}$, while the amorphous film shows a hole mobility up to $1.9 \times 10^{-3} \mathrm{~cm}^{2} / \mathrm{V} \mathrm{s}$ and an electron mobility up to $2.7 \times 10^{-3} \mathrm{~cm}^{2} / \mathrm{V} \mathrm{s}$ for fields from $1.6 \times 10^{5}$ to 7.1 $\times 10^{5} \mathrm{~V} / \mathrm{cm}$. Under a similar field, the aligned film has room-temperature mobilities more than an order of magnitude ( $\sim 14-17$ times) larger than those of the amorphous film. Such mobility enhancement is independent of the type of carriers, indicating similar effects of molecular ordering on hole and electron transport. It is worth mentioning that while similar enhancement of hole mobility through meto AIP license or copyright; see http://apl.aip.org/apl/copyright.jsp 

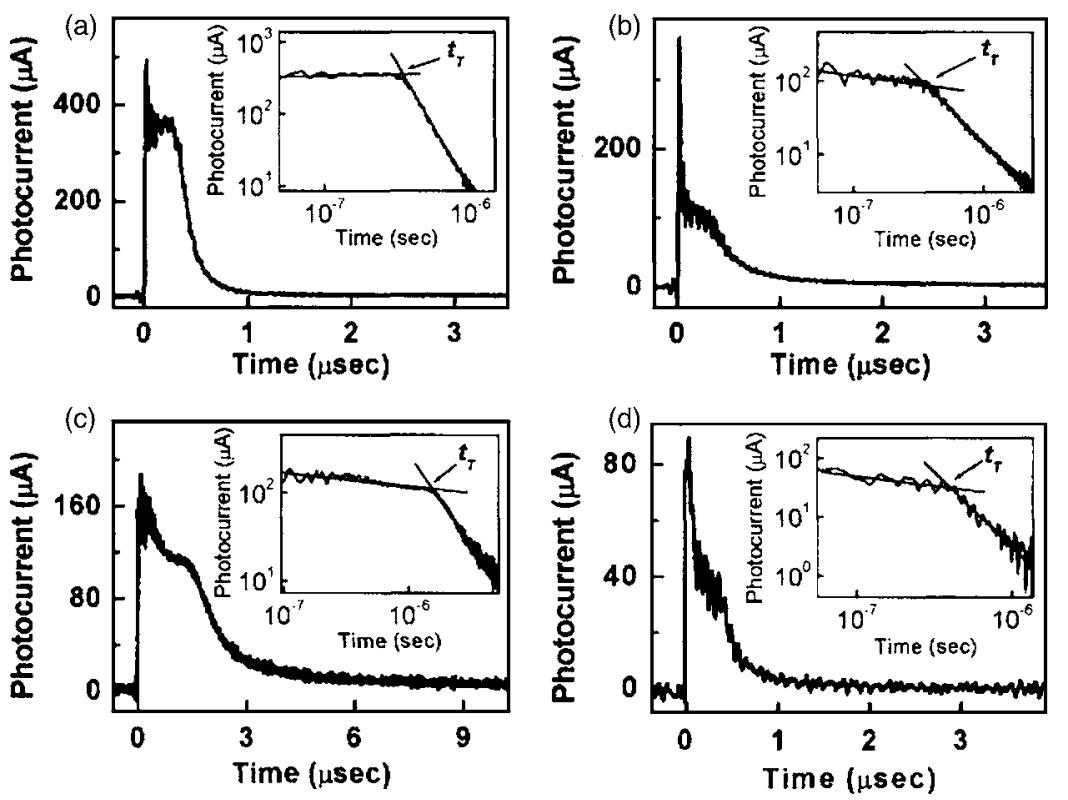

FIG. 3. Representative TOF transients for E4: (a) amorphous film $(1.28 \mu \mathrm{m})$, hole, $E=3.1 \times 10^{5} \mathrm{~V} / \mathrm{cm}$; (b) amorphous film $(1.28 \mu \mathrm{m})$, electron, $E=2.3 \times 10^{5}$ $\mathrm{V} / \mathrm{cm}$; (c) aligned liquid-crystal glass $(10 \mu \mathrm{m})$, hole, $E=6 \times 10^{4} \mathrm{~V} / \mathrm{cm}$; and (d) aligned liquid-crystal glass $(10 \mu \mathrm{m})$, electron, $E=2 \times 10^{5} \mathrm{~V} / \mathrm{cm}$. Insets of (a) $-(\mathrm{d})$ are double logarithmic plots of (a)-(d), respectively. sophase alignment was also previously reported in polyfluorene films, ${ }^{10}$ yet nondispersive electron transport was not observed in polyfluorenes in either the amorphous phase or the glassy LC phase. Such difference is most probably associated with well-defined structures, absence of chain defects, and ease of purification of oligomers in comparison with polymers, as electron transport in organic materials usually are more sensitive to extrinsic issues. ${ }^{20}$ Our results therefore have the significant implication that enhancing carrier mobility in organic solid films through mesophase-mediated molecular alignment is effective for both holes and electrons, as long as extrinsic issues are carefully excluded.

In summary, we investigate the formation of aligned LC glasses of an oligofluorene and perform comparative studies of carrier-transport properties of the oligofluorene in both amorphous films and glassy LC films. With mesophase-
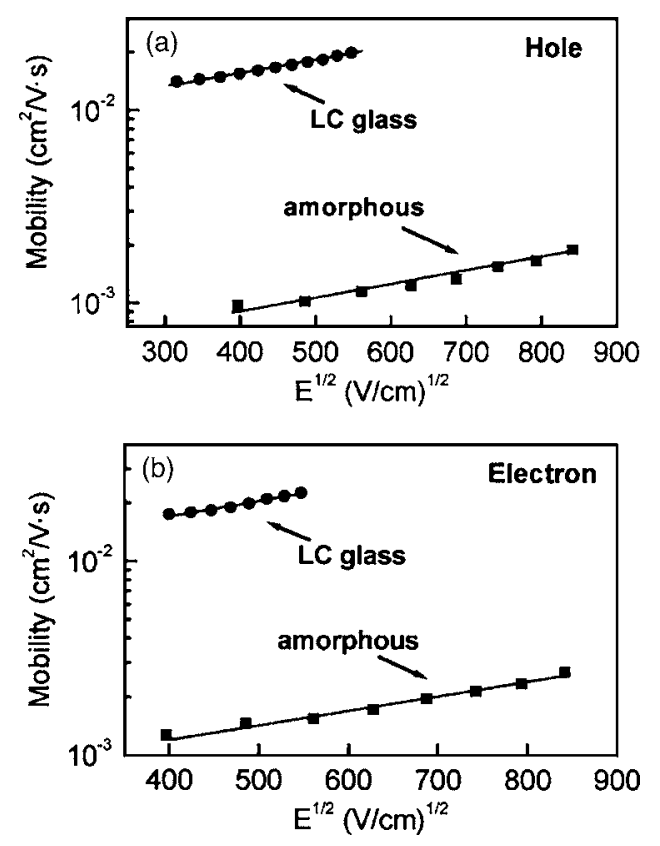

FIG. 4. (a) Hole mobilities and (b) electron mobilities vs $E^{1 / 2}$ for $\mathbf{E} \mathbf{4}$ in the amorphous state and in the aligned liquid-crystal glass. mediated molecular alignment, the bipolar carrier-transport capability of oligofluorene solid films is enhanced by more than an order of magnitude, giving a hole mobility up to $2.0 \times 10^{-2} \mathrm{~cm}^{2} / \mathrm{V} \mathrm{s}$ and an electron mobility up to 2.3 $\times 10^{-2} \mathrm{~cm}^{2} / \mathrm{V} \mathrm{s}$.

The authors would like to acknowledge financial support from National Science Council of Republic of China and Evervictory Acutech Corp. The authors also would like to thank Dr. Ching-Chao Chang of HannStar Display Corp. for stimulating discussions.

${ }^{1}$ P. Strohriegal and J. G. Grazulevicius, Adv. Mater. (Weinheim, Ger.) 14, 1439 (2002).

${ }^{2}$ Y. Shirota, J. Mater. Chem. 10, 1 (2000).

${ }^{3}$ L. B. Schein, Phys. Rev. B 15, 1024 (1977).

${ }^{4}$ R. A. Street, D. Knipp, and A. R. Völkel, Appl. Phys. Lett. 80, 1658 (2002).

${ }^{5}$ K. Naito and A. Miura, J. Phys. Chem. 97, 6240 (1993).

${ }^{6}$ D. Adam, F. Closs, T. Frey, D. Funhoff, D. Haarer, H. Ringsdorf, P. Schuhmacher, and K. Siemensmeyer, Phys. Rev. Lett. 70, 457 (1993).

${ }^{7}$ M. Funahashi and J.-I. Hanna, Phys. Rev. Lett. 78, 2184 (1997).

${ }^{8}$ M. Funahashi and J.-I. Hanna, Appl. Phys. Lett. 71, 602 (1997).

${ }^{9}$ S. Méry, D. Haristoy, J.-F. Nicoud, D. Guillon, S. Diele, H. Monobe, and S. Shimizu, J. Mater. Chem. 12, 37 (2002).

${ }^{10}$ M. Redecker, D. D. C. Bradley, M. Inbasekaran, and E. P. Woo, Appl. Phys. Lett. 74, 1400 (1999).

${ }^{11}$ S. R. Farrar, A. E. A. Contoret, M. O'Neill and J. E. Nicholls, G. J. Richards, and S. M. Kelly, Phys. Rev. B 66, 125107 (2002).

${ }^{12}$ H. Sirringhaus, R. J. Wilson, R. H. Friend, M Inbasekaran, W. Wu, E. P. Woo, M. Grell, and D. D. C. Bradley, Appl. Phys. Lett. 77, 406 (2000).

${ }^{13}$ Y. Geng, S. W. Culligan, A. Trajkovska, J. U. Wallace, and S. H. Chen, Chem. Mater. 15, 542 (2003).

${ }^{14}$ Y. Geng, A. Trajkovska, D. Katsis, J. J. Ou, S. W. Culligan, and S. H. Chen, J. Am. Chem. Soc. 124, 8337 (2002).

${ }^{15}$ C.-C. Wu, T.-L. Liu, W.-Y. Hung, Y.-T. Lin, K.-T. Wong, R.-T. Chen, Y.-M. Chen, and Y.-Y. Chien, J. Am. Chem. Soc. 125, 3710 (2003).

${ }^{16}$ C.-C. Wu, T.-L. Liu, Y.-T. Lin, W.-Y. Hung, T.-H. Ke, K.-T. Wong, and T.-C. Chao, Appl. Phys. Lett. 85, 1172 (2004).

${ }^{17}$ P. M. Borsenberger and D. S. Weiss, Organic Photoreceptors for Imaging Systems (Marcel Dekker, New York, 1993).

${ }^{18}$ H. Bässler, Philos. Mag. B 65, 795 (1992).

${ }^{19}$ H. Bässler, Phys. Status Solidi B 15, 175 (1993).

${ }^{20}$ G. G. Malliaras, Y. Shen, D. H. Dunlap, H. Murata, and Z. H. Kafafi, Appl. Phys. Lett. 79, 2582 (2001). 